\title{
Age-Related Differences in Neuropathic Pain Behavior and Spinal Microglial Activity after L5 Spinal Nerve Ligation in Male Rats
}

\author{
Hossein Zeinali ${ }^{1}$, Homa Manaheji2 ${ }^{\text {, }}$, Jalal Zaringhalam ${ }^{3}$, Zahra Bahari ${ }^{1}$, Samad Nazemi $^{4}$, Mehdi Sadeghi $^{5}$ \\ 1. Department of Physiology, School of Medicine, Shahid Beheshti University of Medical Sciences, Tehran, Iran \\ 2. Neurophysiology Research Center, Shahid Beheshti University of Medical Sciences, Tehran, Iran. \\ 3. Neuroscience Research Center, Shahid Beheshti University of Medical Sciences, Tehran, Iran. \\ 4. Department of Physiology, School of Medicine, Sabzevar University of Medical Sciences, Sabzevar, Iran. \\ 5. Department of Physiology, Faculty of Medicine, Boushehr University of Medical Sciences, Boushehr, Iran.
}

đtration: Zeinali, H., Manaheji, H., Zaringhalam, J., Bahari, Z., Nazemi, S., \& Sadeghi, S. (2016). Age-related differences in neuropathic pain behavior and spinal microglial activity after L5 spinal nerve ligation in male rats. Basic and Clinical Neuroscience, 7(3), $203-212$. http://dx.doi.org/10.15412/J.BCN.03070305

: http://dx.doi.org/10.15412/J.BCN.03070305

Article info:

Received: 16 March 2015

First Revision: 03 April 2015

Accepted: 06 September 2015
Key Words:

Age- related,

Hyperalgesia, Allodynia,

Ibal

\begin{abstract}
A B S T RA C T
Introduction: Several studies have reported the involvement of age-related changes in the development of neuropathic pain behaviors. However, limited data are available on the role of age in establishing and maintaining chronic neuropathic pain after peripheral nerve injury.

Methods: In the present study, we examined age-related neuropathic behavior among rats in 4 age groups: pups (4 weeks old; weight, 60-80 g), juvenile rats (6 weeks old; weight, 120-140 g), and mature rats (10-12 weeks old; weight, 200-250 g). Because the exact contribution of spinal microglia and its association with the development of neuropathic pain remains unknown, we also evaluated the expression of spinal Ibal, a microglial marker, by using western blotting before and 5 days after spinal nerve ligation (SNL) as well as after the daily IP administration of minocycline $(30 \mathrm{mg} / \mathrm{kg})$.

Results: Our results showed that SNL-induced mechanical allodynia but not thermal hyperalgesia in mature rats but not in pups $(\mathrm{P}<0.05$ and $\mathrm{P}<0.01$, respectively). The expression of spinal Ibal in the juvenile rats was significantly lower than that in pups and mature rats $(\mathrm{P}<0.01)$. Moreover, administration of minocycline decreased the expression of spinal Ibal in the pup rats more than in juvenile rats $(\mathrm{P}<0.001)$ and in the juvenile rats more than in the mature rats $(\mathrm{P}<0.05)$.
\end{abstract}

Conclusion: These data suggest that the development of neuropathic behaviors and microglial activation after SNL could be age dependent.

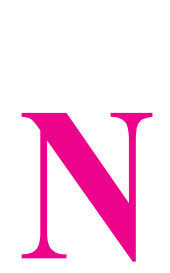

\section{Introduction}

europathic pain is characterized by spontaneous pain, hyperalgesia, and allodynia. To date, no effective treatment is available for neuropathic pain. The mechanisms underlying this debilitating disorder are not completely understood. It has been suggested that development of chronic pain may be influenced by age (Resnick, Levy, \& Jannetta, 1998; Rokyta et al., 2008) i.e. changes in neurochemical and anatomical organization occur with advanced age (Mills, Hains, Johnson,

* Corresponding Author:

Homa Manaheji, $P h D$

Address: Neurophysiology Research Center, Shahid Beheshti University of Medical Sciences, Tehran, Iran.

Tel: +98 (21) 22439971

E-mail:manahejih@sbmu.ac.ir_hshardimanaheji@yahoo.com 
\& Hulsebosch, 2001; Bennett, Everhart, \& Hulsebosch, 2000). However, chronic pain may be influenced by many factors such as sex, age, diet, and genetic differences (Mills \& Hulsebosch, 2002; Hains, Willis, \& Hulsebosch, 2002; Mogil, 2006), and the exact effect of age differences on the development of neuropathic pain is unclear. Some studies have reported that the lower incidence of neuropathic pain in children compared to adults (Drew, Siddall, \& Duggan, 2001; Gwak, Hains, Johnson, \& Hulsebosch, 2003).

Postnatal development of nociceptive pathways and central mechanisms of neuropathic pain in the first weeks of life are completely functional in juvenile animals (Nakamura \& Bregman, 2001; Hains, Everhart, Fullwood, 2003). A recent study indicated that peripheral nerve injury does not induce mechanical allodynia in rats aged less than 3 weeks. Interestingly, pain symptoms are not observed even when these rats (nerve-injured) reach the age at which an injury normally evokes mechanical allodynia (Stuesse, Cruce, Lovell, McBurney, \& Crisp, 2000; Gwak, et al., 2003).

Microglial cells play a pivotal role in the development of neuropathic pain (Tsuda, Inoue, \& Salter, 2005). Spinal cord microglia is strongly activated after nerve injury in the adult rats (Nakamura \& Bregman, 2001). Spared nerve injury (SNI) evokes dorsal horn microglial activation 5 days after operation. Unlike a feeble microglia response in young animals at day 1 of SNI, there was strong astrocytes response, something that was not seen in adults. These results reveals interesting points about the microglia response to nerve injury which may show the lack neuropathic allodynia in young animals (VegaAvelaira, Moss, \& Fitzgerald, 2007; Guasti et al., 2009; Padi \& Kulkarni, 2008).

Some studies suggest that activated microglia exhibit the increased the expression of microglial marker Iba1 (Mitchell \& Boss, 2002; Mogil, Chesler, Wilson, Juraska, \& Sternberg, 2000). Glial cell proliferation is rarely detected under normal conditions. However, robust microglial proliferation has been observed in different neuropathic pain models (Mogil et al., 1999; Shir, Ratner, Raja, Campbell, \& Seltzer, 1998). Some studies have shown that preemptive, intraperitoneal, or the intrathecal application of minocycline induces antiallodynic and antihyperalgesic effects in neuropathic pain (Kar \& Quirion, 1995; Magnone, Rossolini, Piantanelli, Migani, 2000). Therefore, the first aim of the present study was to evaluate the effect of age differences on the initiation and development of neuropathic pain behavior and the second aim was to evaluate the effect of age differences on spinal microglial activity in spinal nerve ligated rats.

\section{Methods}

\subsection{Animals}

Male Wistar rats belonging to 3 age groups (pup rats: 4 weeks old, 60-80 g; juvenile rats: 6 weeks, 120-140g; and mature rats: 10-12 weeks, 200-250 g) were caged with 12:12 h light/ dark cycles and free access to food and water ad libitum. Animal experiments were performed according to the National Institute of Health (NIH) guidelines for the Care and Use of Laboratory Animals and were approved by the Animal Ethics Committee of Shahid Beheshti University of Medical Sciences, Tehran, Iran (194/-90/3/18; 2011).

\subsection{Surgical preparation}

Neuropathic pain was induced according to the method described by Kim and Chung (1992). Animals were anesthetized using pentobarbital sodium (60 mg/ $\mathrm{kg}$, intraperitoneal). The left paraspinal muscles were separated at the $\mathrm{L}_{4}-\mathrm{S}_{2}$ levels, and the left transverse process of the $\mathrm{L}_{6}$ vertebra was removed. The $\mathrm{L}_{5}$ spinal nerve was isolated and tightly ligated using a silk thread $(6 / 0)$, and the distal part of the ligature was transected. The wound was closed later with $3 / 0$ silk threads. In the control sham group, the same surgical procedure was performed, except that the left $\mathrm{L}_{5}$ spinal nerve was not ligated and transected instead. Only those animals that showed no sign of motor deficiency (not limping) were considered for further experiments. To study the agerelated role of microglia, minocycline $(10$ and $30 \mathrm{mg}$ / $\mathrm{kg}$ ) (Sigma, USA) dissolved in saline was immediately injected intraperitoneally after the operation and then daily up to day 5 after the surgery in all the rats.

\subsection{Behavioral study}

Von Frey and Hargreaves tests were used to confirm the successful induction of neuropathy. Sham-operated $(n=8)$ and nerve-injured rats $(n=8)$ were examined for the development of mechanical allodynia and thermal hyperalgesia 1 day before and 2, 5, and 7 days after neuropathy.

\subsubsection{Mechanical allodynia}

To measure paw withdrawal threshold to mechanical stimuli, each animal was placed in a Plexiglas chamber with a metal mesh floor. Thresholds determined by the up-down method (Chaplan, Bach, Progel, Chung, \& 

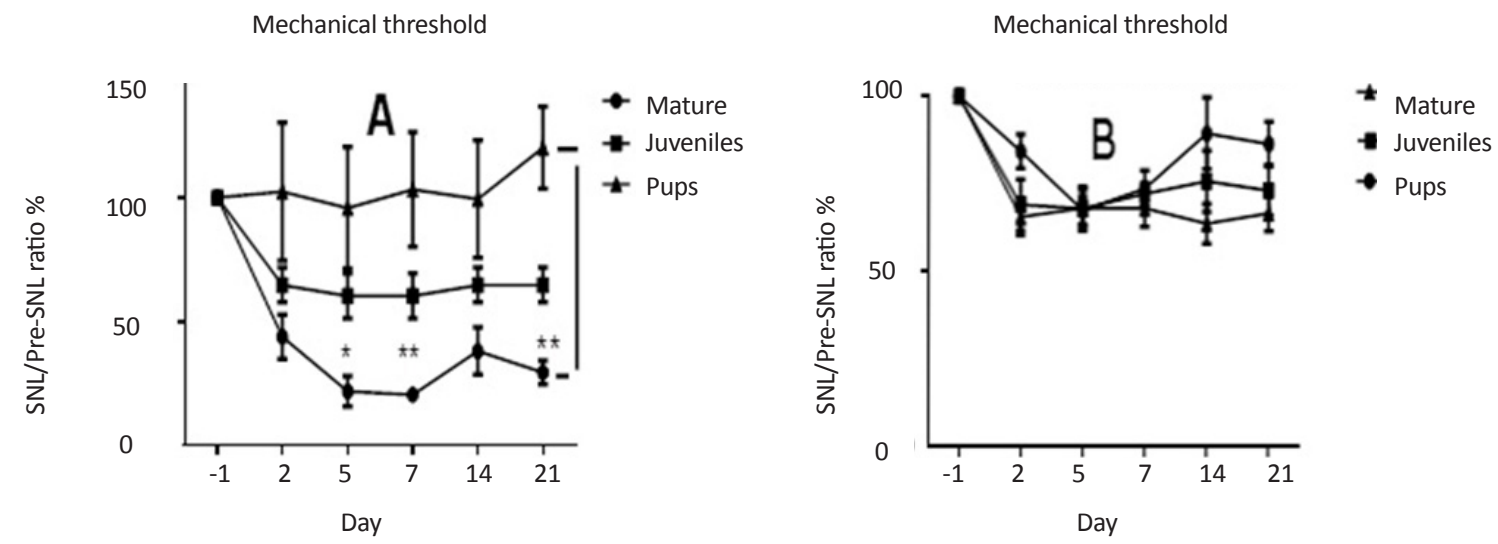

NEUR:SCIENCE

Figure 1. Comparison of mechanical (A) and thermal (B) threshold ratios (SNL/preSNL), 1 day before and 2, 5, 7 and 21 days after SNL in pup, juvenile, and mature rats. SNL decreased mechanical threshold ratio (postSNL/preSNL) in mature rats than pup and juvenile ones. This ratio was significantly lower in mature rats than pup ones on day $5(\mathrm{~F}(1,14)=29.95, \mathrm{P}<0.05)$ until day $21(\mathrm{~F}(4,56)=37.36, \mathrm{P}<0.01)$ postinjury. (2-way ANOVA repeated measures, $\mathrm{n}=6,{ }^{*} \mathrm{P}<0.05$ and ${ }^{* *} \mathrm{P}<0.01$ ). No significant differences were observed between pup and juvenile rats, juvenile and mature rats. Moreover, the thermal threshold ratio showed no significant difference between the 3 different age groups.

Yaksh, 1994). Mechanical stimuli were applied perpendicular to the plantar surface at the proximal half of the third and fourth toes of the injured hindpaw by using a set of Von Frey monofilaments (Stoelting Co, Wood Dale, IL, USA). The evoked hindpaw (left hind paw) was stimulated by using 1 of the 7 Von Frey filaments, with logarithmically incremental stiffness $(2,4,6,8,15$, 26 , and 60 (force) g). Each monofilament was applied to the left hindpaw for approximately 2-3 seconds with sufficient force to buckle the filament.
Each filament was applied 3 times at approximately 5 minutes intervals. Each application started using an 8 (force) g stimulus, that increased or decreased in intensity until the obvious paw withdrawal was observed. Quick withdrawal or licking of the paw in response to stimulation by a particular filament was considered as a positive response. In the presence of a response, the next filament was applied with a feeble force. In the absence of a response, the next filament was applied with an in-
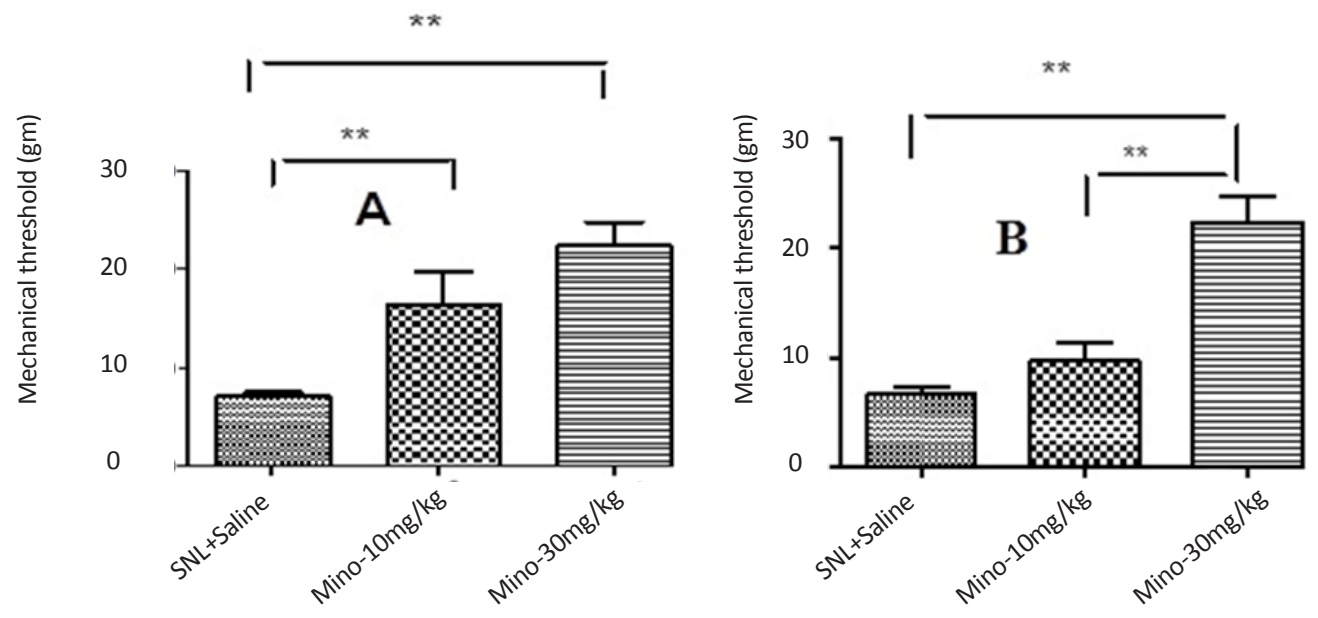

NEUR:SCIENCE

Figure 2. The effect of different doses of minocycline 5 days after SNL plus intraperitoneal administration of minocycline immediately and daily after SNL up to $5^{\text {th }}$ day after SNL on threshold of mechanical allodynia in juvenile (A) and mature (B) rats (Unpaired t-test, $\left.\mathrm{n}=6,{ }^{* *} \mathrm{P}<0.01\right)$. Administration of minocycline $(10,30 \mathrm{mg} / \mathrm{kg})$ significantly increased the threshold of mechanical allodynia in juvenile rats. However, only minocycline $(30 \mathrm{mg} / \mathrm{kg})$ significantly increased the threshold of mechanical allodynia in mature rats compared to the sham group. Since the pup rats showed no significant changes in mechanical allodynia, they omitted from this experiment. 
tense force. Paw withdrawal reaction in at least 2 out of 3 trials was considered as a positive response.

\subsubsection{Thermal hyperalgesia}

Thermal hyperalgesia was assessed as paw withdrawal latency (PWL) by using Hargreaves test. All tests were performed between 8 and 12 AM. The rats were placed in a clear plastic container on an elevated floor made of clear, heat-tempered glass (Plantar Test, Ugo Basile, Italy). After 15 minutes of habituation, a radiant heat source $(50 \mathrm{~W}$ halogen reflector bulbs with intensity controlled by a constant voltage source) was focused on the plantar surface of the ipsilateral and contralateral hindpaws (Nazemi, Manaheji, Zaringhalam, Sadeghi, \& Haghparast, 2012 ). Each paw was tested 5 times with 5 minutes intervals, and the average value of PWL for 5 consecutive tests was recorded. The cut-off time in the absence of a response was 33 seconds to avoid tissue damage. The Hargreaves test confirmed the successful induction of neuropathy before the administration of drugs or a vehicle.

\subsection{Western blotting}

To study the spinal microglial marker Iba1 expression, rats were killed under isoflurane anesthesia, and the lumbar (L5-L6) region of the spinal cord was removed. Tissue samples were homogenized (Brinkmann Polytron Homogenizer, $20000 \mathrm{rpm}, 30 \mathrm{~s})$ in RIPA buffer $(50 \mathrm{mM}$ Tris- $\mathrm{HCl}$ ( $\mathrm{pH} 7.5$ ), $150 \mathrm{mM} \mathrm{NaCl}, 1 \mathrm{mM}$ ethylenediaminetetraacetic acid, 1\% NP40, 0.5\% sodium dodecyl sulfate (SDS), $1 \mathrm{mM}$ sodium orthovanadate, $2.5 \mu \mathrm{g} / \mathrm{ml}$ aprotinin, $2 \mu \mathrm{g} / \mathrm{mL}$ leupeptin, and $2 \mu \mathrm{g} / \mathrm{mL}$ pepstatin A) and were cleared by centrifugation $\left(10000 \times \mathrm{g}\right.$ at $15^{\circ} \mathrm{C}$ for $10 \mathrm{~min})$. Protein concentration in the supernatant was determined using Bradford assay (Bradford, 1976).

Samples containing $60 \mu \mathrm{g}$ of protein were heated for 8 minutes at $99^{\circ} \mathrm{C}$ in loading buffer (4\% SDS, $25 \mathrm{mM}$ Tris- $\mathrm{HCl}$ (pH 6.8), 5\% glycerol, 0.5\% 2-mercaptoethanol, and $0.01 \%$ bromophenol blue) and resolved by SDS polyacrylamide gel electrophoresis on $10 \%$ polyacrylamide gels ( $120 \mathrm{~V}$ for $60 \mathrm{~min}$ ). After electrophoresis, the proteins were electrophoretically transferred to PVDF membranes (Millipore, Bedford, MA) by using miniprotein II (Bio-Rad) at $100 \mathrm{~V}$ for 85 minutes. Nonspecific binding sites were blocked using $2 \%$ blocking buffer ( $0.2 \%$ Aurora Blocking Reagent) in Tris-buffered saline (TBS) with $0.1 \%$ Tween 20 (TBST) for 90 minutes at $24^{\circ} \mathrm{C}$. Mouse monoclonal anti-Ibal (1:2000 dilution; Abcam, Cambridge, UK) was used to detect the level of microglial cell activation.
Blots were incubated overnight with primary antibodies at $4^{\circ} \mathrm{C}$ in a blocking buffer. After three 10 -minutes washing with TBST, the blots were incubated with an appropriate peroxidase-conjugated secondary antibody in blocking buffer (1:10000 dilution) for 1 hour at room temperature. After another three 10-minute washes with TBST and another wash in TBS, the immunoreactivity of the proteins on the membrane was visualized using chemiluminescence detection system (ECL Advance; Amersham). The blots were stripped (stripping buffer: $100 \mu \mathrm{M}$ 2-mercaptoethanol, 2\% SDS, and $62.5 \mathrm{mM}$ Tris, $\mathrm{pH}$ 6.7) at $50^{\circ} \mathrm{C}$ for 30 minutes and reprobed using a mouse polyclonal primary antibody against $\beta$-actin (1:5000 dilution; cell signaling) as a loading control.

The results were quantified by performing densitometric scanning of the films. Data analysis was performed using ImageJ (V1.41, NIH, USA) after background subtraction. Each experiment was replicated 3 times with new groups of rats.

\subsection{Statistical analysis}

All data are presented as mean \pm standard error of the mean (SEM). In the behavioral study, the data were analyzed using 1- and 2-way repeated measures ANOVA, followed by Bonferroni post hoc test. Graphical and statistical analyses were performed using GraphPad Prism Version 5.0 (GraphPad Prism Software, San Diego, CA, USA).

\section{Results}

3.1. Time profile of mechanical allodynia and thermal hyperalgesia among rats in 3 age groups

SNL decreased mechanical threshold ratio (postSNL/ preSNL) in mature rats compared to pup and juvenile ones. This ratio was significantly lower in mature rats than pup ones on day $5(\mathrm{~F}(1,14)=29.95, \mathrm{P}<0.05)$ and continued until 21 days $(\mathrm{F}(4,56)=37.36, \mathrm{P}<0.01)$ postinjury (Figure 1-A). No significant differences were observed between pup rats and juvenile ones, or between juvenile rats and mature ones. Moreover, the thermal threshold ratio showed no significant difference among 3 age groups of rats (Figure 1-B).

\subsection{Effect of minocycline on SNL-induced allo- dynia}

Administration of minocycline (10 and $30 \mathrm{mg} / \mathrm{kg}$ ) increased significantly the threshold of mechanical allodynia in juvenile rats (Figure 2-A); however, only mino- 


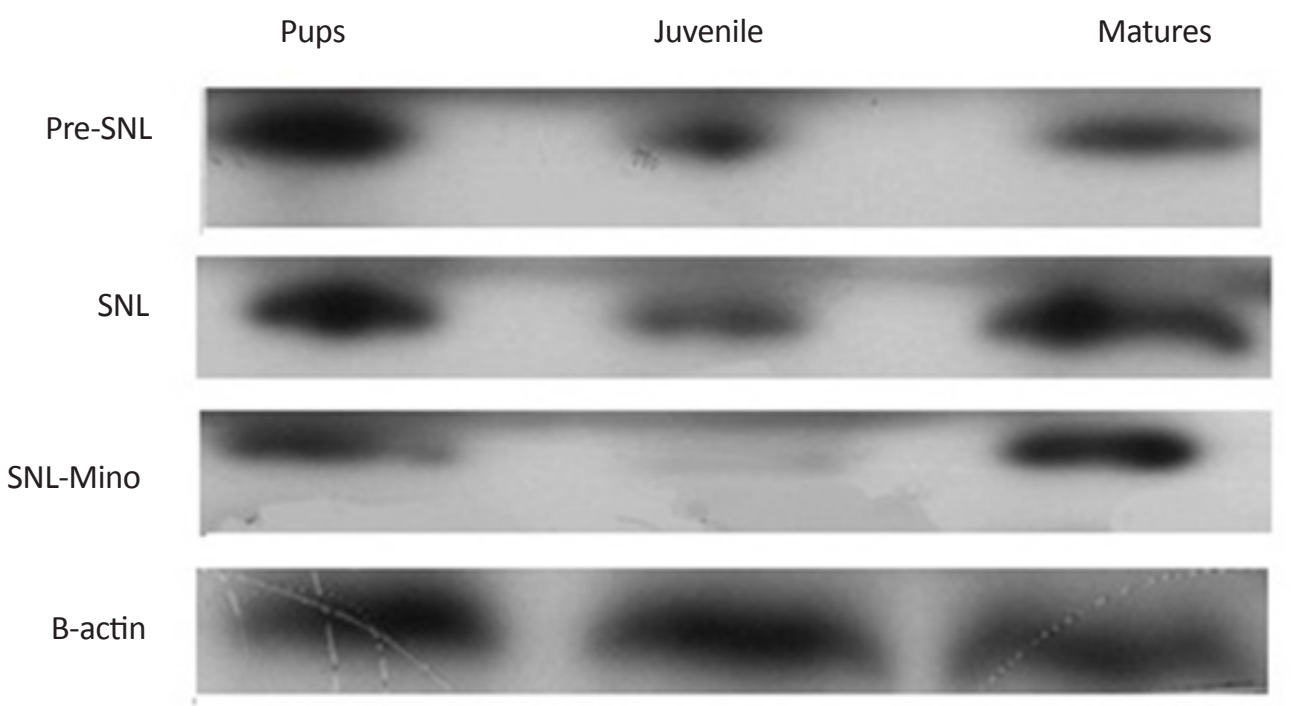

NEUR:SIENCE

Figure 3. Evaluation of Iba1 expression by western blot among 3 different age groups before SNL (Pre SNL), on the $5^{\text {th }}$ day after SNL (post SNL), and on the $5^{\text {th }}$ day after SNL plus administration of minocycline $(30 \mathrm{mg} / \mathrm{kg})$ immediately and daily after SNL up to $5^{\text {th }}$ day after SNL. SNL induced the overexpression of spinal Iba1 in all different age groups. In contrast, administration of minocycline decreased significantly the expression of Iba1 in all age groups, especially in juvenile rats.

cycline $(30 \mathrm{mg} / \mathrm{kg})$ increased significantly threshold of mechanical allodynia in mature rats compared to sham groups (Figure 2-B). Since the pup rats showed no significant changes in mechanical allodynia, they omitted from this experiment.

\subsection{Effect of SNL and minocycline on Iba1 ex- pression}

SNL induced the overexpression of spinal Iba1 in all group of the rats. In contrast, administration of minocycline significantly decreased the expression of Iba1 in all different age groups, especially juvenile group (Figures 3,4$)$.

\subsection{Comparison of Iba1 expression ratio among rats belonging to different age groups}

Expression of Iba1 was significantly lower in juvenile rats than pup and mature ones (Figure 5-A). The ratio of Iba1 expression (postSNL/preSNL) significantly increased after SNL; However, it was lower in juvenile group compared to pup and mature rats (Figure 5-B). Moreover, after minocycline administration, the expression ratio was significantly lower in mature rats than pup and juvenile ones (Figure 5-C).

\section{Discussion}

In this study, SNL resulted in higher induction of mechanical allodynia in juvenile and mature rats com- pared to pup ones. Comparison of PWL ratio of thermal stimuli showed no significant differences among rats in these 3 age groups. Our results are consistent with those of Fitzgerald study, which showed that brachial plexus injury induced devastating chronic neuropathic pain in adults but minimal chronic pain in neonates (Fitzgerald, 2005). Facial neuralgia is rare in children (Fitzgerald \& MacDermott, 2005; Grazzi, Usai, \& Rigamonti, 2005) and adults; its early symptoms manifest during childhood, which do not need any therapy (Howard, Walker, Mota, \& Fitzgerald, 2005). It is generally accepted that perinatal and early postnatal periods play a crucial role in ontogenetic development (Rokyta et al., 2008; Wei et al., 2010). Postnatal development of nociceptive pathways and central mechanisms of neuropathic pain in the first few weeks of life are completely functional in juvenile animals (Hains, Everhart, Fullwood, 2003; Nakamura \& Bregman, 2001).

Prenatal or neonatal stress may disturb the development of the nociceptive system and induce long-term behavioral changes that persist during adulthood. However, some type of neuropathic pain cannot be induced in the first two weeks of postnatal period. Therefore, a mature nervous system is required to observe the development of pathological behaviors (Rokyta et al., 2008; Howard, 2003). Apparently, different mechanisms exist for allodynia and hyperalgesia development and these behaviors are age dependent (Wei et al., 2010). These events play a pivotal role in age-related neuropathic 

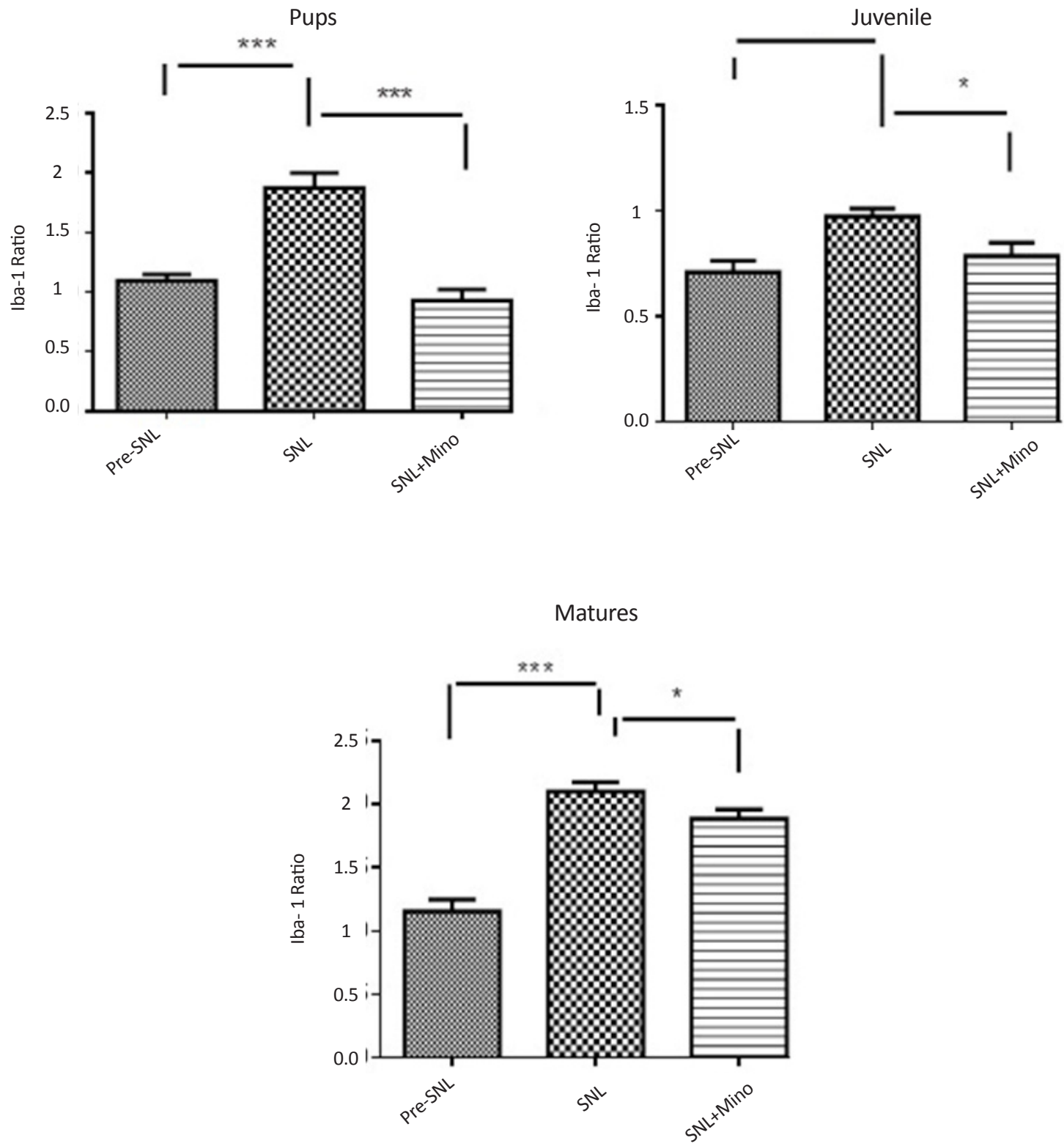

NEUR:SCIENCE

Figure 4. Spinal Iba1 expression before SNL (preSNL), 5 days after SNL (postSNL) and on the $5^{\text {th }}$ day after SNL plus intraperitoneal administration of minocycline $(30 \mathrm{mg} / \mathrm{kg}$ ) immediately and daily after SNL in 3 different age groups (Unpaired t-test, $\mathrm{n}=6,{ }^{*} \mathrm{P}<0.05$, ${ }^{* *} \mathrm{P}<0.01$, and $\left.{ }^{* * *} \mathrm{P}<0.001\right)$. Iba1 ratio: Iba1/ $\beta$-actin. SNL induced overexpression of spinal Iba1 in all age groups of the rats. In contrast, administration of minocycline significantly decreased the expression of Iba1 in all different age groups

pain after peripheral nerve injury (Wen, Tan, Cheng, Liu, \& Ji, 2011; Echeverry, Shi, \& Zhang, 2008).

Previous studies have indicated that microglial activation in the spinal cord and higher centers is one of the main causes of increasing sensitivity to pain transmission after a nerve injury (Ledeboer et al., 2005; Suter, Wen, Decosterd, \& Ji, 2007). Also, a relationship exists between nerve damage, microglial activation, and neuropathic pain (Anand \& Birch, 2002; Grazzi, Usai, \& Rigamonti, 2005). In the present study, SNL signifi- cantly increased spinal Iba1 expression in all rats. However, allodynia and hyperalgesia were not significant in pups after SNL.

Several studies have also confirmed the association of hyperalgesic state and prolife ratio with activation of microglia in the spinal cord and higher centers. It has been shown that lack of allodynia in nerve-injured pups may arise from immature microglial response (Fitzgerald, 2005). Furthermore, CCI and SNL neuropathy activate spinal microglia in rats belonging to all age groups 

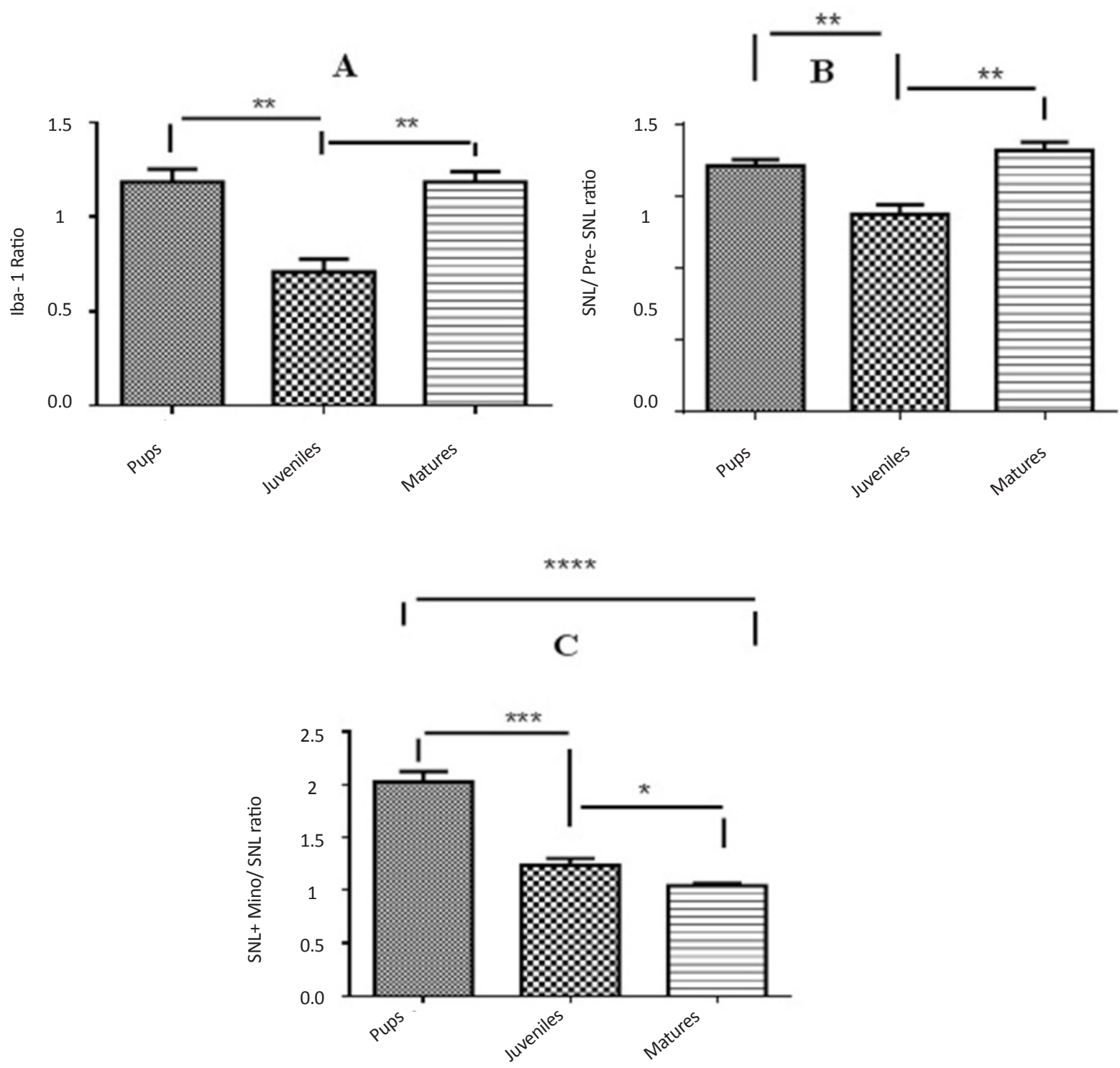

NEUR:SCIENCE

Figure 5. Comparison of Iba1 expression ratios among different age groups before SNL (preSNL), 5 days after SNL (postSNL) and after SNL plus administration of minocycline $\left(30 \mathrm{mg} / \mathrm{kg}\right.$ ) (Unpaired t-test, $\mathrm{n}=6,{ }^{*} \mathrm{P}<0.05$, ${ }^{* *} \mathrm{P}<0.01$, and $\left.{ }^{* * *} \mathrm{P}<0.001\right)$. Iba1 ratio: Iba1/ $\beta$-actin. The expression of Iba1 was significantly lower in juvenile rats than pup and mature ones (A). The ratio of Iba1 expression (post SNL/pre SNL) significantly increased after SNL; However, it was lower in juvenile rats than pup and mature rats (B). Moreover, after minocycline administration, the expression ratio was significantly lower in mature rats than pup and juvenile rats $(C)$.

(Guasti et al., 2009; Padi \& Kulkarni, 2008). Moreover, a study has shown microglial activation after bilateral damage to the spinal nerve with PLS in young male Wistar rats (weight, 120-150 g) (Ledeboer et al., 2005).

Several studies have also confirmed association of hyperalgesic state and proliferation with activation of microglia in the spinal cord and higher centers. Glial cells such as microglia are also critical for the development of neuropathic pain. These studies also showed that increasing neuronal release of mediators such as ATP, glutamate, and neuropeptides after nerve injury induces glial cell activity (Grazzi, Usai, \& Rigamonti, 2005; Zhuo, Wu, \& Wu, 2011). However, the potential factors that may contribute to microglial activation after nerve injury are still unclear. It has been shown that daily administration of minocycline $(10,20$, or $40 \mathrm{mg} / \mathrm{kg}$ intraperitoneally) beginning 1 hour before nerve transection decreases mechanical hyperalgesia and allodynia, with a maximum inhibitory effect at the dose of 20 and 40 
$\mathrm{mg} / \mathrm{kg}$. The inhibitory effect of minocycline on the developement of hyperalgesia and allodynia is due to the inhibition of microglial activation at L5 lumbar spinal cord in nerve injured animals. These results indicate that inhibition of microglial activation may prevent the occurrence of neuronal hypersensitivity in neuropathic pain (Raghavendra, Tanga, \& DeLeo, 2003).

In our study, after-SNL/before-SNL ratio of spinal Iba1 expression was different among three age groups, i.e. higher in pup and mature rats. It seems that SNLinduced expression of spinal Ibal is age dependent, but the relationship between age and the level of expression is different. The effects of age on Ibal expression and microglial activity before nerve injury are unknown. It has indicated that in adult rats, SNI provokes strong microglia response at 5 and astrocyte at 7 days after surgery. Unlike adults, in young animals, SNI lead to poor response in the microglia and strong in astrocytes at day 1 . These results provide interesting facts about the microglia response to nerve injury which may show the lack neuropathic allodynia in young animals, something that was not seen in adults. Also SNI-induced Iba1 overexpression is age dependent but the relationship between age and Ibal expression is different to some extent (Vega-Avelaira, Moss, \& Fitzgerald, 2007; Guasti et al., 2009; Padi \& Kulkarni, 2008).

In our study, daily administration of minocycline (microglial inhibitor) decreased allodynia in juvenile and mature rats. However, the decrease was higher in juvenile rats than in mature rats and the effect of minocycline decreased with an increase in age. It seems that age is a pivotal factor for the activation, induction, and maintenance of neuropathic pain after SNL. Consistent with the present study, Mika, Osikowicz, Makuch, \& Przewlocka (2007) showed that minocycline (20 and 30 $\mathrm{mg} / \mathrm{kg}$ ) prevented the activation of microglia and symptoms of neuropathic pain in rats after SNI.

The present study showed that mechanical allodynia (not thermal hyperalgesia) develops in parallel to the expression of spinal Iba1, which is age dependent. Moreover, the effect of minocycline on allodynia and spinal Ibal expression is age dependent too.

\section{Acknowledgements}

This project was supported by the Neuroscience Research Center of Shahid Beheshti University of Medical Sciences, Tehran, Iran.

\section{References}

Anand, P., \& Birch, R. (2002). Restoration of sensory function and lack of long-term chronic pain syndromes after brachial plexus injury in human neonates. Brain, 125(1), 113-122.

Bennett, A. D., Everhart, A. W., \& Hulsebosch, C. E. (2000). Intrathecal administration of an NMDA or a non-NMDA receptor antagonist reduces mechanical but not thermal allodynia in a rodent model of chronic central pain after spinal cord injury. Brain Research, 859(1), 72-82.

Bradford, M. M. (1976). A dye binding assay for protein. Analytical Biochemistry, 72, 248-254.

Chaplan, S., Bach, F., Progel, J., Chung, J., \& Yaksh, T. (1994). Quantitative assessment of tactile allodynia in the rat paw. Journal of Neuroscience Methods, 53(1), 55-63.

Drew, G. M., Siddall, P. J., \& Duggan, A. W. (2001). Responses of spinal neurones to cutaneous and dorsal root stimuli in rats with mechanical allodynia after contusive spinal cord injury. Brain Research, 893(1), 59-69.

Echeverry, S., Shi, X. Q., \& Zhang, J. ( 2008). Characterization of cell proliferation in rat spinal cord following peripheral nerve injury and the relationship with neuropathic pain. Pain, 135(1-2), 37-47.

Fitzgerald, M. (2005). The development of nociceptive circuits. Nature Review Neuroscience, 6(7), 507-520.

Fitzgerald, M., \& MacDermott, A. (2005). The development of pain systems. In L. Ede (Ed.), Neurobiology of Pain: Molecular and Cellular Neurobiology (pp. 207-238). UK: Oxford University Press.

Grazzi, L., Usai, S., \& Rigamonti, A. (2005). Facial pain in children and adolescents. Neurological Sciences, 26(2), 101-103.

Guasti, L., Richardson, D., Jhaveri, M., Eldeeb, K., Barrett, D., Elphick, M. R., et al. (2009). Minocycline treatment inhibits microglial activation and alters spinal levels of endocannabinoids in a rat model of neuropathic pain. Molecular Pain, 5(35), 1-10.

Gwak, Y. S., Hains, B. C., Johnson, K. M., \& Hulsebosch, C. E. ( 2004). Effect of age at time of spinal cord injury on behavioral outcomes in rat. Journal of Neurotroma, 21(8), 983-93.

Gwak, Y.S., Nam, T. S., Paik, K. S., Hulsebosch, C. E., \& Leem, J. W. (2003). Attenuation of mechanical hyperalgesia following spinal cord injury by administration of antibodies to nerve growth factor in the rat. Neuroscience Letter, 336(2), 117-120.

Hains, B. C., Willis,W. D., \& Hulsebosch C. E. (2003). Serotonin receptors 5-HT1A and 5-HT3 reduce hyperexcitability of dorsal horn neurons after chronic spinal cord hemisection injury in rat. Experimental Brain Research, 149(2), 174-186.

Hains, B. C., Everhart, A. W., Fullwood, S. D., \& Hulsebosch, C. E. (2002). Changes in serotonin, serotonin transporter expression and serotonin denervation supersensitivity: involvement in chronic central pain after spinal hemisection in the rat. Experimental Neurology, 175(2), 347-362.

Howard, R. F. (2003). Current status of pain management in children. Journal of the American Medical Association, 290(18), 2464-2469. 
Howard, R. F., Walker, S. M., Mota, P. M., \& Fitzgerald, M. (2005). The ontogeny of neuropathic pain: Postnatal onset of mechanical allodynia in rat spared nerve injury (SNI) and chronic constriction injury (CCI) models. Pain, 115(3), 382389.

Kar, S., \& Quirion, R. (1995). Neuropeptide receptors in developing and adult rat spinal cord: An in vitro quantitative autoradiography study of calcitonin gene-related peptide, neurokinins, mu-opioid, galanin, somatostatin, neurotensin and vasoactive intestinal polypeptide receptors. Journal of Comparative Neurology, 354(2), 253-281.

Kim, S. H., \& Chung, J. M. (1992). An experimental model for peripheral neuropathy produced by segmental spinal nerve ligation in the rat. Pain, 50(3), 355-363.

Ledeboer, A., Sloane, E. M., Milligan, E. D., Frank, M. G., Mahony, J. H., \& Maier, S. F. (2005). Minocycline attenuates mechanical allodynia and proinflammatory cytokine expression in rat models of pain facilitation. Pain, 115(1-2), 71-83.

Magnone, M. C., Rossolini, G., Piantanelli, L. \& Migani, P. (2000). Neurochemical parameters of the main neurotransmission systems in aging mice. Archives of Gerontology and Geriatric, 30(3), 269-279.

Mika, J., Osikowicz, M., Makuch, W., \& Przewlocka, B. (2007). Minocycline and pentoxifylline attenuate allodynia and hyperalgesia and potentiate the effects of morphine in rat and mouse models of neuropathic pain. European Journal of Pharmacology, 560(2-3), 142-149.

Mills, C. D., Hains, B. C., Johnson, K. M., \& Hulsebosch, C. E. (2001). Strain and model differences in behavioral outcomes after spinal cord injury in rat. Journal of Neurotrauma, 18(8), 743-756.

Mills, C. D., \& Hulsebosch, C. E. (2002). Increased expression of metabotropic glutamate receptor subtype 1 on spinothalamic tract neurons following spinal cord injury in the rat. Neuroscience Letter, 319(2), 59-62.

Mitchell, A. \& Boss, B. J. (2002). Adverse effects of pain on the nervous systems of newborns and young children: A review of the literature. Journal of Neuroscience Nursing, 34(5), 228 236 .

Mogil, J. S. (2006). Sex, gender and pain. In F. Cervero \& T. S Jensen (Eds.), Pain: Handbook of clinical neurology (Vol. 81, pp. 325-343). Amstredam: Elsevier, B.V.

Mogil, J. S., Chesler, E.J., Wilson, S. G., Juraska, J. M., \& Sternberg, W. F. (2000). Sex differences in thermal nociception and morphine antinociception in rodents depend on genotype. Neuroscience Biobehavoral Review, 24(3), 375-89.

Mogil, J. S., Wilson, S. G., Bon, K., Lee, S. E., Chung, K., \& Raber, P., et al. (1999). Heritability of nociception II.Types' of nociception revealed by genetic correlation analysis. Pain, 80(1), 83-93

Nakamura, M., \& Bregman, B. S. (2001). Differences in neurotrophic factor gene expression profiles between neonate and adult rat spinal cord after injury. Experimental Neurology, 169(2), 407-415

Nazemi, S., Manaheji, H., Zaringhalam, J., Sadeghi, M., \& Haghparast, A. (2012). Post-injury repeated administrations of minocycline improve the antinociceptive effect of morphine in chronic constriction injury model of neuropathic pain in rat. Pharmacology, Biochemistry and Behavior, 102, 520525.

Padi, S. S., \& Kulkarni, S. K. (2008). Minocycline prevents the development of neuropathic pain, but not acute pain: Possible anti-inflammatory and antioxidant mechanisms. European Journal of Pharmacology, 601(1-3), 79-87.

Raghavendra, V., Tanga. F., \& DeLeo, J. A. (2003). Inhibition of microglial activation attenuates the development but not existing hypersensitivity in a rat model of neuropathy. Journal of Pharmacology and Experimental Theraputhic, 306(2), 624-630.

Resnick, D. K., Levy, E. I., \& Jannetta, P. J. (1998). Microvascular decompression for pediatric onset trigeminal neuralgia. Neurosurgery, 43(4), 804-807.

Rokyta, R., Yamamotova, A., Slamberova, R., Frankek, M., Vaculin, S., \& Hruba, L., et al. (2008). Prenatal and perinatal factors influencing nociception, addiction and behavior during ontogenetic development. Physiological Research, 57(3), 79-88.

Shir, Y., Ratner, A., Raja, S. N., Campbell, J. N., \& Seltzer, Z. (1998). Neuropathic pain following partial nerve injury in rats is suppressed by dietary soy. Neuroscience Letter, 240(2), 73-76

Stuesse, S. L., Cruce, W. L., Lovell, J. A, McBurney, D. L., \& Crisp, T. (2000). Microglial proliferation in the spinal cord of aged rats with a sciatic nerve injury. Neuroscience Letter, 287(2), 121-124.

Suter, M. R., Wen, Y. R., Decosterd, I., \& Ji, R. R. (2007). Do glial cells control pain? Neuron Glia Biology, 3(3), 255-268.

Tsuda, M., Inoue, K., \& Salter, M. W.( 2005). Neuropathic pain and spinal microglia: a big problem from molecules in "small" glia. Trends in Neurosciences, 28(2), 101-107.

Vega-Avelaira, D., Moss, A., \& Fitzgerald, M. (2007). Age-related changes in the spinal cord microglial and astrocytic response profile to nerve injury. Brain, Behavior, and Immunity, 21(5), 617-623.

Wei, H., Hao, B., Huang, J. L., Ma, A. N., Li, X. Y., \& Wang, Y. $X .(2010)$. Intrathecal administration of a gap junction decoupler, an inhibitor of $\mathrm{Na}^{+} \mathrm{K}^{+} 2 \mathrm{Cl}^{-}$cotransporter 1 , or a $\mathrm{GABA}_{\mathrm{A}}$ receptor agonist attenuates mechanical pain hypersensitivity induced by REM sleep deprivation in the rat. Pharmacology Biochemistry and Behavior, 97(2), 377-383.

Wen, Y. R., Tan, P. H., Cheng, J. K., Liu, Y. C., \& Ji, R. R. (2011). Microglia: a promising target for treating neuropathic and postoperative pain, and morphine tolerance. Journal of the Formosan Medical Association, 110(8), 487-494.

Zhuo, M., Wu, G., \& Wu, L. J. (2011). Neuronal and microglial mechanisms of neuropathic pain. Molecular Brain, 4(31), 1-12 
